\title{
Orexin-A inhibits capsaicin-induced changes in cyclooxygenase-2 and brain-derived neurotrophic factor expression in trigeminal nucleus caudalis of rats
}

\author{
${ }^{1}$ Department of Biology, Faculty of Sciences, Shahid Bahonar University of Kerman, ${ }^{2}$ Endodontology Research Center, Kerman \\ University of Medical Sciences, ${ }^{3}$ Laboratory of Molecular Neuroscience, Neuroscience Research Center, Institute of \\ Neuropharmacology, Kerman University of Medical Sciences, ${ }^{4}$ Pathology and Stem Cell Research Center, Department of \\ Pathology, Afzalipour Kerman University of Medical Science, Kerman, Iran \\ Razieh Kooshki', Mehdi Abbasnejad', Saeed Esmaeili Mahani', \\ Maryam Raoof ${ }^{2,3}$, Mohammad Mehdi Moeini Aghtaei ${ }^{4}$, and Shahriar Dabiri ${ }^{4}$
}

Background: The trigeminal nucleus caudalis (Vc) is a primary central site for trigeminal transmitting. Noxious stimulation of the trigeminal nociceptors alters the central synaptic releases and neural expression of some inflammatory and trophic agents. Orexin-A and the orexin 1 receptor (OX1R) are expressed in pain pathways including trigeminal pain transmission. However, the the mechanism(s) underling orexin-A effects on trigeminal pain modulation have not been fully clarified.

Methods: Trigeminal pain was induced by subcutaneous injection of capsaicin in the upper lip in rats. The effect of trigeminal pain on cyclooxygenase-2 (COX-2) and brain-derived neurotrophic factor (BDNF) expression in the Vc of animals was determined by immunofluorescence. Subsequently, OX1R agonist (orexin-A) and antagonist (SB-334867-A) was administrated in the Vc to investigate the possible roles of the Vc OX1R on changes in COX-2 and BDNF levels following pain induction.

Results: The data indicated an increase in COX-2 and decrease in BDNF immuno-reactivity in the Vc of capsaicin, and capsaicin- pretreated with SB-334867-A $(80 \mathrm{nM})$, groups of rat. However, the effect of capsaicin on COX-2 and BDNF expressions was reversed by a Vc microinjection of orexin-A (100 pM).

Conclusions: Overall, the present data reveals that orexin-A can attenuate capsaicin-induced trigeminal pain through the modulation of pain effects on COX-2 and BDNF expressions in the Vc of rats. (Korean J Pain 2018; 31: 174-82)

Key Words: Brain-derived neurotrophic factor (BDRF); Capsaicin; Cyclooxygenase 2 (COX 2); Orexin-A; Orexin receptor antagonists; Orofacial pain; Rats; Pain measurement; Pain perception; Trigeminal caudal nucleus; Trigeminal neuralgia.

Received March 30, 2018. Revised June 3, 2018. Accepted June 12, 2018

Correspondence to: Maryam Raoof

Endodontology Research Center, Kerman University of Medical Sciences, Kerman 7616913555, Iran

Tel: +98-9133416108, Fax: +98-3412118073, E-mail: Maryam.raoof@gmail.com

() This is an open-access article distributed under the terms of the Creative Commons Attribution Non-Commercial License (http:// creativecommons.org/licenses/by-nc/4.0/), which permits unrestricted non-commercial use, distribution, and reproduction in any medium, provided the original work is properly cited.

Copyright (c) The Korean Pain Society, 2018 


\section{INTRODUCTION}

Due to different types of treatments and resources, trigeminal pain is one of the most distressing health concerns around the world. It's evoked by a sensory stimulus of the trigeminal branches in the head, face, and mouth structures through the initial processing in the trigeminal nucleus caudalis $(\mathrm{Vc})[1,2]$.

Noxious stimulation and peripheral inflammation lead to a subsequent release of inflammatory mediators from the presynaptic trigeminal neuronal terminals. Induced cyclooxygenase-2 (COX -2$)$ protein expression in the trigeminal tissue has also been reported. COX-2 is an inducible enzyme and commonly considered as a source of prostaglandin, induced by inflammatory stimuli. It has been demonstrated that COX-2 overexpression can induce hypersensitivity and excitability of nociceptor sensory terminals $[3,4]$.

Brain-derived neurotrophic factor (BDNF), a key neurotrophin within the central nervous system, plays critical roles in neural survival and plasticity [5]. Various distributional studies of BDNF and BDNF receptors also indicated that they are strongly expressed in nociceptive fibers in the spinal cord and dorsal root ganglion, as well as the trigeminal ganglion and nuclei [6,7]. Interestingly, it has been shown that trigeminal pains can alter the synaptic releases of BDNF [8]. However, the modulating effects of BDNF on nociceptive pathways, and BDNF expression in inflamed tissues, are controversial.

Anti-nociceptive and pro-nociceptive, as well as different levels of BDNF expressions, in an inflammatory condition, influence trkB and trkC neurotrophin receptor agonists on thermal nociception in behavioral and electrophysiological studies $[7,9,10]$.

Orexin peptides (orexin-A and orexin-B), originally found in the lateral hypothalamus, stimulate target cells through two G-protein-coupled receptors, the orexin 1 and orexin 2 receptors, OX1R and OX2R, respectively. Orexin-A binds equally to both receptors, while orexin-B has a better affinity for OX2R [11]. Because of their broad central distribution, orexin peptides are involved in the regulation of various physiological functions, including pain and cognitive processes [12,13]. Specifically, orexin A has a modulating role on trigeminal pain transmission. It has been shown that the posterior hypothalamic and intravenous administration of orexin-A can suppress trigeminal nerve firing in rats [12,14]. Intra-Vc administration of orexin-A can also attenuate capsaicin-induced orofacial pain [15].

However, the underlying mechanisms of orexin- $\mathrm{A}$ inhibitory effect on trigeminal pain have not yet been fully investigated. In the present study, the effect of intra-Vc administration of OX1R agonist and antagonist on orofacial pain-induced changes in the COX 2 and BDNF expression was investigated in rats.

\section{MATERIALS AND METHODS}

\section{Animals}

Twenty-four adult male Wistar rats (230-270 g) were used. The rats were housed in a temperature-controlled room at $23 \pm 1^{\circ} \mathrm{C}$, with a standard $12 \mathrm{~h}$ light/dark cycle, and received food and water ad libitum. The experimental protocol was approved by the ethics committee of Kerman University of Medical Sciences, Kerman, Iran. All efforts were made to minimize distress.

\section{Surgery}

The rats were deeply anesthetized by an intraperitoneal (i.p) injection of a ketamine $(100 \mathrm{mg} / \mathrm{kg})$ and xylazine (10 $\mathrm{mg} / \mathrm{kg}$ ) mixture. The heads of the animals were fixed in a stereotaxic apparatus and a stainless steel guide cannula was implanted bilaterally in the skull $(13.8 \mathrm{~mm}$ posterior to the bregma, $2.2 \mathrm{~mm}$ lateral from the midline and 7.5 $\mathrm{mm}$ deep to the cortical surface above the $\mathrm{Vc}$ ) according to the Paxinos and Watson atlas of the rat brain [16]. The cannulas were attached to them using dental acrylic cement. The cannulas were then tightly closed by two stainless-steel screws.

After surgery, all the animals had at least 1 week of recovery before the microinjections. The location of the cannula in relation to skull coordinates, and a coronal section through the Vc, adapted from the atlas of Paxinos and Watson, are presented in Fig. 1. At the end of the behavioral experiment, methylene blue was injected via the guide cannula to verify the precise location of the cannula. If the cannula was misplaced, the rat's data was omitted from the analysis. 

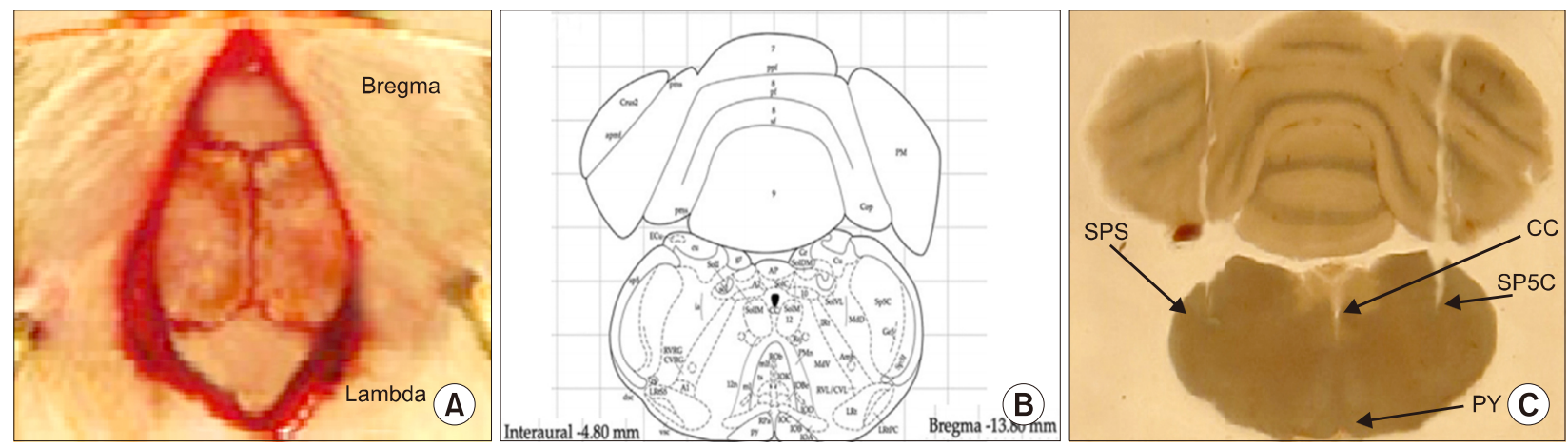

Fig. 1. The cannula insertion and the microinjection procedures steps $(A)$ and a coronal section through trigeminal nucleus caudalis ( $\mathrm{V}$ ) adapted from the atlas of Paxinos and Watson (B and C). SP5C: spinal trigeminal nucleus caudalis, SP5: spinal trigeminal tract, CC: central canal, PY: pyramidal tract.

\section{Drugs}

Capsaicin was dissolved in Tween 80 and ethanol solution (1 ethanol, 1 Tween 80, 8 distilled water). Orexin-A and SB-334867-A (Tocris Bioscience, Bristol, UK) were dissolved in distilled water and dimethyl sulfoxide, respectively.

\section{Microinjection}

The rats were kindly handled and the microinjections were performed without any anesthesia. Orexin-A and SB$334867-\mathrm{A}$ were administered into the $\mathrm{Vc}$ via the guide cannula (22-gauge) using an injection needle (27-gauge) connected by a polyethylene tube to a $1 \mu$ l Hamilton microsyringe. The injection needle was inserted $1 \mathrm{~mm}$ beyond the tip of the guide cannula. Infusions were delivered in a total injection volume of $2 \mu \mathrm{l}(1 \mu \mathrm{l}$ per side) over a period of $1 \mathrm{~m}$. The injector was allowed to remain in the guide cannula for $30 \mathrm{~s}$ to avoid blocking.

\section{Experimental groups}

Four groups of rats $(n=6)$ were used: a normal control group (intact), a capsaicin-treated group which received intra-lip capsaicin injection with no cannulation; and orexin-A and SB-334867-A pretreated group, which received intra a Vc microinjection of orexin-A (100 pM) and SB-334867-A (80 nM) twenty minutes before capsaicin administration.

The dose of drugs and the number of animal per group were determined from previous studies [15,17]. Besides, our previous study indicated that intra- $\mathrm{Vc}$ administration of
orexin-A vehicle, distilled water, and DMSO as a vehicle of OX1Rs antagonist (SB-334867-A) had no effect on the strictness of capsaicin-induced orofacial pain. So in the current molecular trial we didn't evaluate vehicle groups [15].

The schematic drawing of the experimental procedures is presented in Fig. 2.

\section{Pain induction}

On the day of the test, rats were taken to the testing room and habituated for $30 \mathrm{~m}$. Trigeminal pain was induced by a subcutaneous injection of capsaicin $(100 \mu \mathrm{g} / 10 \mu \mathrm{l})$ with a 30-gauge hypodermic needle into the upper lip $20 \mathrm{~m}$ following the Vc administration of drugs.

Afterward, the animals were placed in a clear Plexiglas test box $(30 \times 30 \times 30 \mathrm{~cm})$, with a mirror placed at a $45^{\circ}$ angle beneath the floor allowing for unbarred observation of the rats.

The nociceptive response was evaluated by measuring the number of seconds of a typical pattern of face rubbing behavior for $40 \mathrm{~m}$, as previously described [15].

\section{Immunofluorescence}

Twenty-four hours after drug administration, the animals were anesthetized by i.p. injection of a ketamine/xylazine mixture (100 and $10 \mathrm{mg} / \mathrm{kg}$, respectively) and perfused transcardially with $0.9 \%$ saline $(100 \mathrm{ml})$ followed by $4 \%$ paraformaldehyde $(500 \mathrm{ml})$.

The brains were post-fixed overnight in $4 \%$ paraformaldehyde. The sections were made from the rostral regions of the trigeminal nucleus caudalis (Vc), $200 \mu \mathrm{m}$ in 
Cannula implantation in the $\mathrm{Vc}$

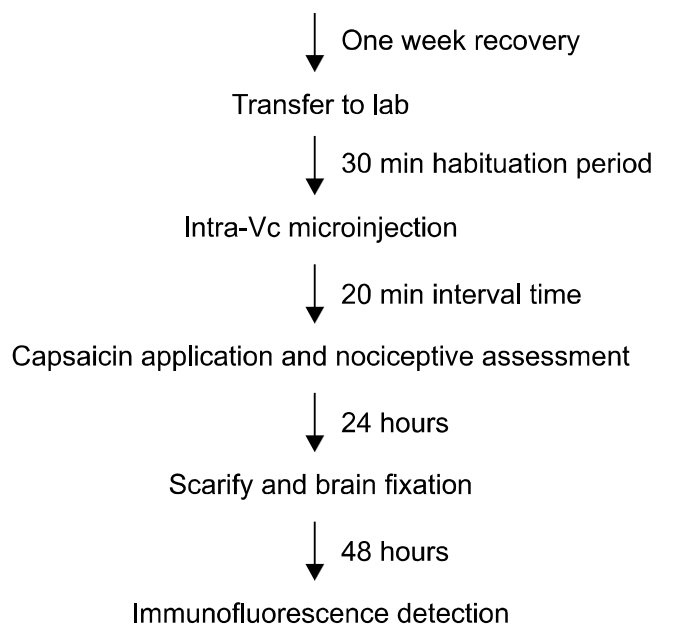

Fig. 2. Schematic drawing of the experimental procedures.

thickness, $2.2 \mathrm{~mm}$ lateral to the midline and $13.8 \mathrm{~mm}$ posterior to the bregma according to the Paxinos and Watson rat brain atlas. The samples were embedded in paraffin and then $2 \mu \mathrm{m}$ sections were prepared from the $\mathrm{Vc}$, containing paraffin blocks and were deparaffinized before immunostaining. The slides were hydrated through 100\%, $90 \%$, $70 \%$, and $50 \%$ ethanol.

The sections then were treated for antigen retrieval using microwave treatment for $30 \mathrm{~m}$ in a citrate buffer $(\mathrm{pH}$ 6.0). They were washed in phosphate-buffer saline (PBS) for $3 \mathrm{~m}$. The slides were dipped in hydrogen peroxide for $10 \mathrm{~m}$ and then exposed to anti-COX-2 or BDNF primary antibodies diluted at 1:500 (Santa Cruz Biotechnology, Inc, Dallas, TX) overnight in a humidity chamber.

The slides were washed in PBS and then incubated for 90 min with goat anti-mouse or goat anti-rabbit IgG-CFL 488 secondary antibodies at a dilution of $1: 1000$ (Santa Cruz Biotechnology, Inc, Dallas, TX) and washed with PBS. The sections were exposed to propidium iodide, at room temperature, and again washed with PBS.

Fluorescence microscopic images were captured with a digital camera, at a magnification of 20 times. All positive COX-2 or BDNF immuno-reactive cells in each $\mathrm{Vc}$ section were manually counted.

\section{Statistical analysis}

All data are presented as means \pm SEM. Statistical analysis was performed using one-way analysis of variance

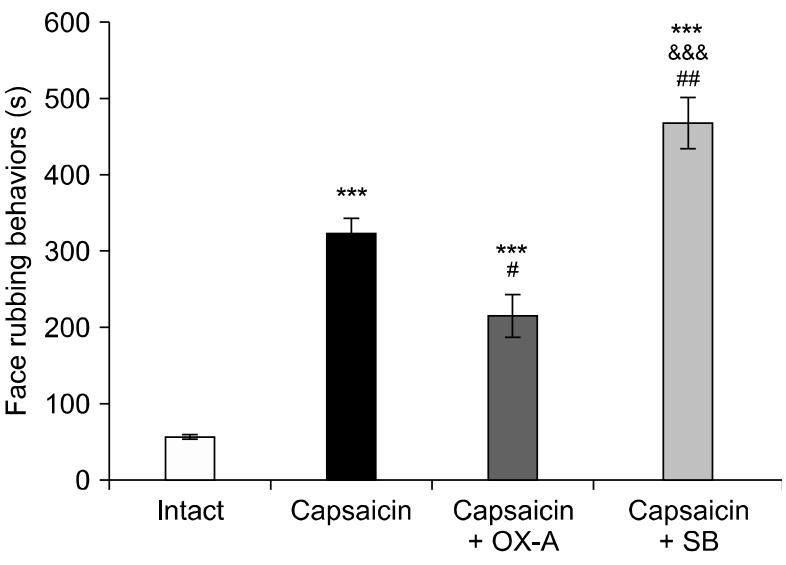

Fig. 3. Effects of intraVc microinjection of orexin-A (100 $\mathrm{pM})$ and SB-334867-A (80 nM) on capsaicin-induced pain behavior. Data are presented as mean \pm SEM $(n=6)$. ${ }^{* *} P<0.001$ versus intact rats, ${ }^{\# \#} P<0.01,{ }^{\#} P<0.05$ versus Caps-treated group, ${ }^{2 \& \&} P<0.001$ versus OX-A plus Caps-treated group. Caps: capsaicin, OXA: orexin-A, SB: SB-334867-A.

(ANOVA) followed by the post-hoc Tukey's test. Differences were considered significant if $P<0.05$.

\section{RESULTS}

\section{Pain assessment}

The capsaicin injection into the upper lip evoked a significant pain response $(P<0.001)$. The cumulative time of pain response was $320.2 \pm 22.63$ s. Intra-Vc administration of orexin-A (100 pM), before the capsaicin injection could decrease the time of nociceptive behavior (214 \pm 229) $(P<0$. 05). However, in capsaicin plus SB-334867-A $(80 \mathrm{nM})$-treated rats, pain response $(468.1 \pm 35.64)$ was exaggerated as compared to the capsaicin-treated animals $(P<0.01)$ (Fig. 3).

2. The effects of Vc administration of OXIRs agonist and antagonist on $\mathrm{COX}-2$ expression in the $\mathrm{Vc}$ of capsaicin-treated rats

As shown in Fig. 4, the number of COX-2 immuno-reactive cells was significantly increased in the capsaicin-treated group $(42.01 \pm 3.14)(P<0.01)$. Pretreatment with orexin-A $(100 \mathrm{pM})$ could diminish the capsaicin effects on COX-2 induction $(P<0.01)$. 
A
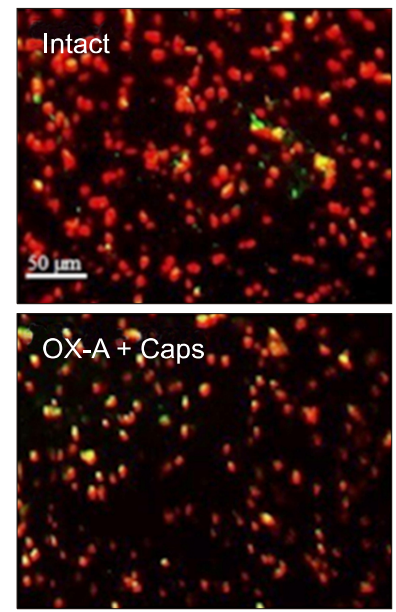
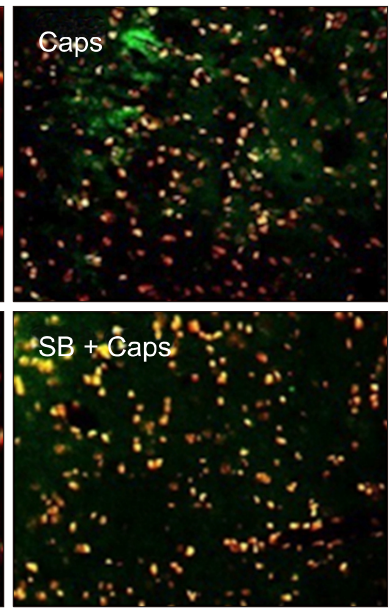

B

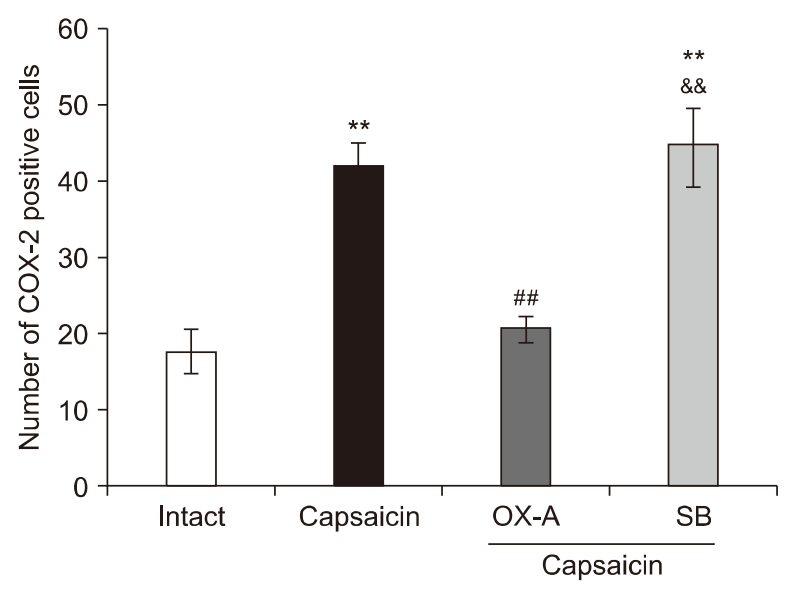

Fig. 4. Almmunofluorescence detection of COX-2 in the $V_{c}$ of rats. $A$ : demonstrating COX-2 staining in $V_{c}$ cells (green in color), propidium iodide (PI) staining to indicate the nucleus of cells (red in color) and the merged of COX-2 and $\mathrm{PI}$ (yellow in color) in intact, capsaicin and capsaicin plus orexin-A (100 pM) or SB-334867-A (80 nM) treated groups. B: statistical comparison of the number of COX-2 positive-cells in $V_{c}$ sections of experimental groups. Data are presented as mean \pm SEM. ${ }^{* \star} P<0.01$, versus intact rats, ${ }^{\# \#} P<0.01$ versus Caps-treated rats, ${ }^{\text {\&\&}} P<0.01$ versus OX-A+ Caps-treated rats. Caps: capsaicin, OX-A: orexin-A, SB: SB-334867-A.

A
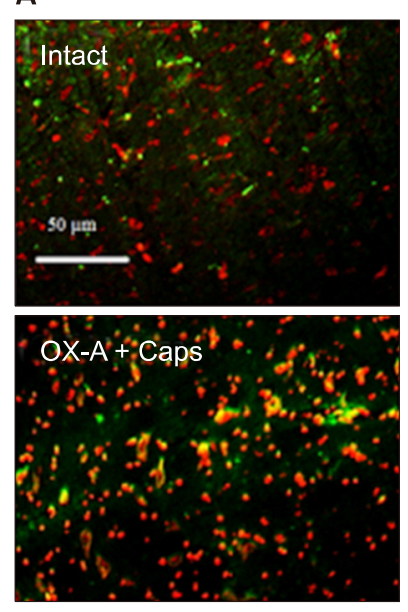
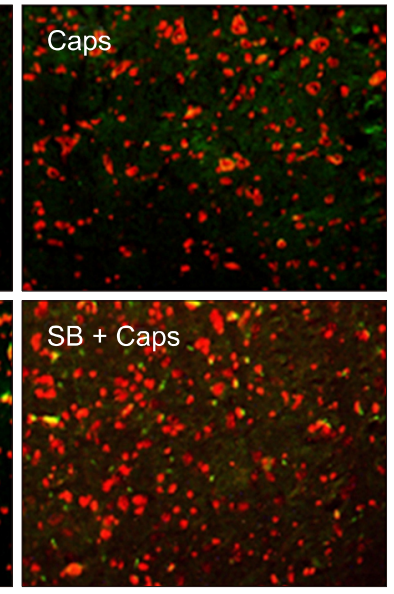

B

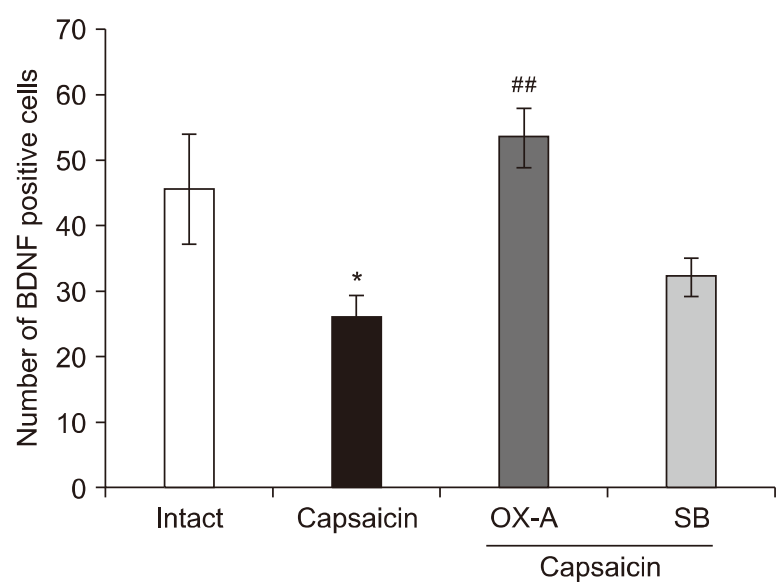

Fig. 5. Almmunofluorescence detection of BDNF in the $V_{c}$ of rats. A: demonstrating BDNF staining in $V_{c}$ cells (green in color), propidium iodide (PI) staining to indicate the nucleus of cells (red in color) and the merged of BDNF and PI (yellow in color) in intact, capsaicin and capsaicin plus orexin-A (100 pM) or SB-334867-A (80 nM) treated groups. B: statistical comparison of the number of BDNF positive-cells in $V_{c}$ sections of experimental groups. Data are presented as mean \pm SEM. ${ }^{*} P<0.05$ versus intact rats, ${ }^{\# \#} P<0.001$ versus Caps-treated rats. Caps: capsaicin, OX-A: orexin-A, SB: SB-334867-A.

Furthermore, in SB-334867-A (80 nM) pretreated rats, the number of positive COX-2 cells (44.64 \pm 5.39) was significantly higher than that in the control and orexin-A injected rats $(P<0.01)$ (Fig. 4).
3. The effects of intra-Vc administration of OXIRs agonist and antagonist on BDNF expression in the $\mathrm{Vc}$ of capsaicin-treated rats

In capsaicin-treated rats, the number of BDNF im- 
muno-reactive cells $(26.31 \pm 3.33)$ was significantly decreased as compared with control rats $(46.02 \pm 8.39)(P<$ 0.05). However, pretreatment with orexin-A (100 pM), before the capsaicin injection, could prevent capsaicin-induced BDNF down-regulation $(P<0.001)$. Additionally, in SB-334867-A (80 nM) pretreated rats, before capsaicin injection, BDNF reactive cells $(32.45 \pm 2.87)$ were significantly decreased as compared to orexin-A injected rats $(P<0.01)$ (Fig. 5).

\section{DISCUSSION}

The present study showed that intra-lip capsaicin injection increased pain behaviors and COX-2 protein levels in the Vc. Interestingly, activation of OXIRs in the Vc could reduce capsaicin-induced pain as well as COX-2 expression. There is growing evidence indicating the role of $\mathrm{COX}-2$ in the modulation of trigeminal pain. Consistent with present results, Geo et al. showed that tooth movement can induce COX-2 expression in the Vc which correlates with an increase in nociceptive response [3]. Moreover, central inhibition of COX-2 has been shown to suppress formalin-induced temporomandibular joint inflammation in rats [18].

Capsaicin, as a potent activator of the TRPV1 channel, is known to promote inflammatory pain via the release of pro-inflammatory neuropeptides such as neurokinin A, substance $\mathrm{P}$, and calcitonin gene related peptide (CGRP) in the trigeminal nerves [19-21]. Interestingly, these neuropeptides have been shown to induce COX-2 expression in the trigeminal nucleus caudalis and trigeminal ganglion [22,23].

In addition, capsaicin can produce hyperalgesia and pain hypersensitivity by alterations in the sensitivity of the peripheral terminals in response to normal and noxious stimulations [24,25]. This situation is generally characterized by overexpression of $\mathrm{COX}-2$ in the spinal and supra-spinal sites $[4,26]$. So, capsaicin-induced COX-2 upregulation may be somewhat correlated with the hyperalgesic and hypersensitivity effects of capsaicin. It may increase the sensitivity of Vc second order neurons which cause the enhancement and facilitation of synaptic transmission and inflammatory events [3].

The data indicated that Vc microinjection of orexin-A can inhibit capsaicin-induced $\mathrm{COX}-2$ up-regulation, supporting the potent anti-inflammatory properties of orexin-A. These results are consistent with some in-vitro and in-vivo studies which indicate the anti-inflammatory and neuro-protective effects of orexin-A [27,28]. It has been shown that Vc administration of orexin-A can diminish capsaicin-induced orofacial inflammation in rats [15]. Moreover, the inhibitory effects of orexin-A on A- and $\mathrm{C}-$ fibers in Vc neurons have been reported $[12,14]$. In contrast, there is just one report that shows that the blockage of orexin receptors can inhibit trigeminal neuron activation via inhibiting the expression of protein kinase A and interleukin 1 beta in the spinal trigeminal nucleus [29].

Activation of the trigeminal nerves and associated glial cells are accompanied with the release of glutamate and various pro-inflammatory peptides that initiate and promote an inflammatory response within the spinal trigeminal nucleus [3]. It has been reported that orexin-A can decrease the expression of tumor necrosis factor alpha in murine microglia cells [30]. Surprisingly, it has been demonstrated that tumor necrosis factor alpha induces orexinergic neural toxicity [31]. In addition, orexin-A can inhibit neurogenic dural vasodilation via the inhibition of CGRP releases from trigeminal neurons [14]. So, the inhibitory effects of orexin-A on COX-2 induction may be partly mediated by suppression of the release of pro-inflammatory peptides from second order neurons in the Vc. However, further studies are needed to elucidate this important issue.

Inflammatory challenges are commonly associated with an increase in pro-apoptotic and oxidant markers which raise the risk of neural loss and deficiency [32]. Specifically, capsaicin has been shown to provoke cell apoptosis [33]. On the other hand, the anti-apoptotic properties of orexin-A have been reported [34]. Butterick et al. demonstrated that orexin-A decreases lipid peroxidation and apoptosis in embryonic rat hypothalamic cells [27]. Moreover, the orexin system has a protective effect against focal ischemia by the modulation of inflammatory responses in mice [35]. In the present study, the decreased expression of capsaicin-evoked COX-2 may be somewhat mediated by the antioxidant and anti-apoptotic properties of orexin-A. However, further studies are needed to explain the detailed role(s) and exact mechanism(s) of orexin-A in this regard.

The data showed that capsaicin-induced trigeminal pain decreased Vc expression of BDNF that was blocked by intra-Vc administration of orexin-A. BDNF as a widespread neurotrophin factor is known to be implicated in the regulation of nociceptive neurotransmission and excitability 
[6]. BDNF, mRNA, and protein are well expressed in primary trigeminal neurons [36]. Moreover, BDNF localization in the second-order sensory neurons of the Vc has been reported [37]. Peripheral irritation of the trigeminal nerves can induce changes in the expression of BDNF in the trigeminal ganglion [38]. Tarsa et al. reported that a greater extent of BDNF expression in the trigeminal ganglion was observed in mice than in rats, following pulp inflammation [39]. However, in-vitro administration of BDNF had no significant effect on capsaicin-induced CGRP releases from cultural trigeminal ganglion neurons [8]. According to data, capsaicin induces loss and probably defects in the transmission of BDNF, might reveal neural damage and toxic effects of capsaicin, which may be reflected by the increase in the number of intracellular reactive oxygen species as well as apoptosis $[40,41]$. These events have been shown to reduce BDNF expression again [42].

The present data also indicated that a Vc microinjection of orexin-A could enhance BDNF levels in the $\mathrm{Vc}_{\mathrm{c}}$ of capsaicin-treated rats. Harada et al. reported that an intra-hypothalamic microinjection of orexin-A can suppress BDNF down-regulation, induced by cerebral ischemic stress and neuronal damage in rats [43]. Moreover, orexins can increase the mRNA expressions of BDNF and neurotrophin-3 in rat primary cortical neuron cultures [44]. The current study seems to establish the possibility that there is a functional interaction between orexin-A and BDNF in the modulation of trigeminal pain. It may reduce the sensitivity of the trigeminal nerves via the alteration of the synaptic releases of sensory neuropeptides that are colocalized with BDNF, containing nociceptive fibers. Interestingly, in-vitro administration of BDNF can reduce release of substance $\mathrm{P}$ from the dorsal horn sensory neurons [45].

Our previous study indicated that orexin-A administration in the $\mathrm{Vc}$ of rats can suppress capsaicin-induced orofacial pain in a dose-dependent manner [15]. So, in the present study the most effective antinociceptive doses of OXIR agonist and antagonist were used to evaluate orexin-A effects on the induction of COX-2 and BDNF in the Vc of rats.

In conclusion, these results suggest that the inhibitory effects of orexin-A on trigeminal pain at least in part may be related to preventing the change in inflammatory and trophic agents including COX-2 and BDNF in Vc neurons. However, further studies need to clarify the exact mecha- nism (s) underlying the effects of orexin-A on trigeminal pain modulation.

\section{ACKNOWLEDGEMENTS}

This study was done through the financial support of Neuroscience Research Center and Shahid Bahonar University, Kerman, Iran.

\section{CONFLICTS OF INTEREST}

The authors declared that they have no conflict to interest.

\section{REFERENCES}

1. Sessle BJ. Neural mechanisms and pathways in craniofacial pain. Can J Neurol Sci 1999; 26 Suppl 3: S7-11.

2. Boggero IA, Rojas-Ramirez MV, de Leeuw R, Carlson CR. Satisfaction with life in orofacial pain disorders: associations and theoretical implications. J Oral Facial Pain Headache 2016; 30: 99-106.

3. Gao Y, Duan YZ. Increased COX2 in the trigeminal nucleus caudalis is involved in orofacial pain induced by experimental tooth movement. Anat Rec (Hoboken) 2010; 293: 485-91.

4. Lee KM, Kang BS, Lee HL, Son SJ, Hwang SH, Kim DS, et al. Spinal NF-kB activation induces COX-2 upregulation and contributes to inflammatory pain hypersensitivity. Eur $J$ Neurosci 2004; 19: 3375-81.

5. Lu Y, Christian K, Lu B. BDNF: a key regulator for protein synthesis-dependent LTP and long-term memory? Neurobiol Learn Mem 2008; 89: 312-23.

6. Merighi A, Salio C, Ghirri A, Lossi L, Ferrini F, Betelli C, et al. BDNF as a pain modulator. Prog Neurobiol 2008; 85: 297-317.

7. Zhu ZW, Friess H, Wang L, Zimmermann A, Büchler MW. Brain-derived neurotrophic factor (BDNF) is upregulated and associated with pain in chronic pancreatitis. Dig Dis Sci 2001; 46: 1633-9.

8. Price TJ, Louria MD, Candelario-Soto D, Dussor GO, Jeske NA, Patwardhan AM, et al. Treatment of trigeminal ganglion neurons in vitro with NGF, GDNF or BDNF: effects on neuronal survival, neurochemical properties and TRPV1mediated neuropeptide secretion. BMC Neurosci 2005; 6: 4.

9. Shu $X Q$, Llinas $A$, Mendell $L M$. Effects of trkB and trkC neurotrophin receptor agonists on thermal nociception: a behavioral and electrophysiological study. Pain 1999; 80 : 463-70.

10. Goshen I, Kreisel T, Ben-Menachem-Zidon O, Licht T, Weidenfeld J, Ben-Hur T, et al. Brain interleukin-1 mediates chronic stress-induced depression in mice via adrenocortical 
activation and hippocampal neurogenesis suppression. Mol Psychiatry 2008; 13: 717-28.

11. Sakurai T, Amemiya A, Ishii M, Matsuzaki I, Chemelli RM, Tanaka H, et al. Orexins and orexin receptors: a family of hypothalamic neuropeptides and $\mathrm{G}$ protein-coupled receptors that regulate feeding behavior. Cell 1998; 92: 573-85.

12. Holland PR, Akerman S, Goadsby PJ. Modulation of nociceptive dural input to the trigeminal nucleus caudalis via activation of the orexin 1 receptor in the rat. Eur $\mathrm{J}$ Neurosci 2006; 24: 2825-33.

13. Akbari E, Motamedi F, Davoodi FG, Noorbakhshnia M, Ghanbarian E. Orexin-1 receptor mediates long-term potentiation in the dentate gyrus area of freely moving rats. Behav Brain Res 2011; 216: 375-80.

14. Holland PR, Akerman S, Goadsby PJ. Orexin 1 receptor activation attenuates neurogenic dural vasodilation in an animal model of trigeminovascular nociception. J Pharmacol Exp Ther 2005; 315: 1380-5.

15. Kooshki R, Abbasnejad M, Esmaeili-Mahani S, Raoof M. The role of trigeminal nucleus caudalis orexin 1 receptors in orofacial pain transmission and in orofacial pain-induced learning and memory impairment in rats. Physiol Behav 2016; 157: 20-7.

16. Paxinos G, Franklin KB. The rat brain in stereotaxic coordinates [electronic resource]. 4th ed. San Diego (CA), Academic Press. 1998.

17. Raoof M, Shakoori A, Kooshki R, Abbasnejad M, Amanpour $S$. The effects of regular exercise on capsaicin-induced pulpal pain and pain-induced changes in passive avoidance learning and memory in rats. Korean J Pain 2017; 30: 258-64.

18. Ahn DK, Choi HS, Yeo SP, Woo YW, Lee MK, Yang GY, et al. Blockade of central cyclooxygenase (COX) pathways enhances the cannabinoid-induced antinociceptive effects on inflammatory temporomandibular joint (TMJ) nociception. Pain 2007; 132: 23-32.

19. Zhou Y, Long H, Ye N, Liao L, Yang X, Jian F, et al. The effect of capsaicin on expression patterns of CGRP in trigeminal ganglion and trigeminal nucleus caudalis following experimental tooth movement in rats. J Appl Oral Sci 2016; 24: 597-606.

20. Quartu M, Serra MP, Boi M, Poddighe L, Picci C, Demontis $\mathrm{R}$, et al. TRPV1 receptor in the human trigeminal ganglion and spinal nucleus: immunohistochemical localization and comparison with the neuropeptides CGRP and SP. J Anat 2016; 229: 755-67.

21. Kistner $K$, Siklosi N, Babes A, Khalil M, Selescu T, Zimmermann $\mathrm{K}$, et al. Systemic desensitization through TRPA1 channels by capsazepine and mustard oil - a novel strategy against inflammation and pain. Sci Rep 2016; 6: 28621.

22. Neeb L, Hellen P, Boehnke C, Hoffmann J, Schuh-Hofer S,
Dirnagl $U$, et al. $\|-1 \beta$ stimulates COX -2 dependent $P G E_{2}$ synthesis and CGRP release in rat trigeminal ganglia cells. PLoS One 2011; 6: e17360.

23. Capuano A, De Corato A, Lisi L, Tringali G, Navarra P, Dello Russo C. Proinflammatory-activated trigeminal satellite cells promote neuronal sensitization: relevance for migraine pathology. Mol Pain 2009; 5: 43.

24. Hood DD, Curry R, Eisenach JC. Intravenous remifentanil produces withdrawal hyperalgesia in volunteers with capsaicin-induced hyperalgesia. Anesth Analg 2003; 97 : 810-5.

25. Iwaoka E, Wang S, Matsuyoshi N, Kogure Y, Aoki S, Yamamoto $S$, et al. Evodiamine suppresses capsaicininduced thermal hyperalgesia through activation and subsequent desensitization of the transient receptor potential V1 channels. J Nat Med 2016; 70: 1-7.

26. Hay C, de Belleroche J. Carrageenan-induced hyperalgesia is associated with increased cyclo-oxygenase-2 expression in spinal cord. Neuroreport 1997; 8: 1249-51.

27. Butterick TA, Nixon JP, Billington CJ, Kotz CM. Orexin A decreases lipid peroxidation and apoptosis in a novel hypothalamic cell model. Neurosci Lett 2012; 524: 30-4.

28. Cheng JK, Chou RC, Hwang LL, Chiou LC. Antiallodynic effects of intrathecal orexins in a rat model of postoperative pain. J Pharmacol Exp Ther 2003; 307: 1065-71.

29. Cady RJ, Denson JE, Sullivan LQ, Durham PL. Dual orexin receptor antagonist 12 inhibits expression of proteins in neurons and glia implicated in peripheral and central sensitization. Neuroscience 2014; 269: 79-92.

30. Xiong X, White RE, Xu L, Yang L, Sun X, Zou B, et al. Mitigation of murine focal cerebral ischemia by the hypocretin/orexin system is associated with reduced inflammation. Stroke 2013; 44: 764-70.

31. Zhan S, Cai GQ, Zheng A, Wang Y, Jia J, Fang $H$, et al. Tumor necrosis factor-alpha regulates the hypocretin system via mRNA degradation and ubiquitination. Biochim Biophys Acta 2011; 1812: 565-71.

32. Leszek J, Barreto GE, Gąsiorowski K, Koutsouraki E, Ávila-Rodrigues M, Aliev G. Inflammatory mechanisms and oxidative stress as key factors responsible for progression of neurodegeneration: role of brain innate immune system. CNS Neurol Disord Drug Targets 2016; 15: 329-36.

33. Raoof M, Esmaeili-Mahani S, Nourzadeh M, Raoof $R$, Abbasnejad M, Amirkhosravi L, et al. Noxious stimulation of the rat tooth pulp may impair learning and memory through the induction of hippocampal apoptosis. J Oral Facial Pain Headache 2015; 29: 390-7.

34. Pasban-Aliabadi H, Esmaeili-Mahani S, Abbasnejad M. Orexin-A protects human neuroblastoma SH-SY5Y cells against 6-hydroxydopamine-induced neurotoxicity: involvement of PKC and PI3K signaling pathways. Rejuvenation Res $2017 ; 20$ : 125-33. 
35. Kitamura E, Hamada J, Kanazawa N, Yonekura J, Masuda $\mathrm{R}$, Sakai $\mathrm{F}$, et al. The effect of orexin-A on the pathological mechanism in the rat focal cerebral ischemia. Neurosci Res 2010; 68: 154-7.

36. Ichikawa H, Yabuuchi T, Jin HW, Terayama R, Yamaai T, Deguchi $T$, et al. Brain-derived neurotrophic factorimmunoreactive primary sensory neurons in the rat trigeminal ganglion and trigeminal sensory nuclei. Brain Res 2006; 1081: 113-8.

37. Buldyrev I, Tanner NM, Hsieh HY, Dodd EG, Nguyen LT, Balkowiec A. Calcitonin gene-related peptide enhances release of native brain-derived neurotrophic factor from trigeminal ganglion neurons. J Neurochem 2006; 99: 1338-50.

38. Behnia A, Zhang L, Charles M, Gold MS. Changes in TrkB-like immunoreactivity in rat trigeminal ganglion after tooth injury. J Endod 2003; 29: 135-40.

39. Tarsa L, Ba ł kowiec-Iskra E, Kratochvil FJ 3rd, Jenkins VK, McLean A, Brown AL, et al. Tooth pulp inflammation increases brain-derived neurotrophic factor expression in rodent trigeminal ganglion neurons. Neuroscience 2010; 167: 1205-15.

40. Lee I, Kim HK, Kim JH, Chung K, Chung JM. The role of reactive oxygen species in capsaicin-induced mechanical hyperalgesia and in the activities of dorsal horn neurons. Pain 2007; 133: 9-17.

41. Aubdool AA, Kodji X, Abdul-Kader N, Heads R, Fernandes ES, Bevan S, et al. TRPA1 activation leads to neurogenic vasodilatation: involvement of reactive oxygen nitrogen species in addition to CGRP and NO. Br J Pharmacol 2016; 173: 2419-33.

42. Kapczinski F, Frey BN, Andreazza AC, Kauer-Sant'Anna M, Cunha AB, Post RM. Increased oxidative stress as a mechanism for decreased BDNF levels in acute manic episodes. Rev Bras Psiquiatr 2008; 30: 243-5.

43. Harada S, Yamazaki Y, Tokuyama S. Orexin-A suppresses postischemic glucose intolerance and neuronal damage through hypothalamic brain-derived neurotrophic factor. J Pharmacol Exp Ther 2013; 344: 276-85.

44. Yamada N, Katsuura G, Tatsuno I, Kawahara S, Ebihara K, Saito $Y$, et al. Orexins increase mRNA expressions of neurotrophin-3 in rat primary cortical neuron cultures. Neurosci Lett 2009; 450: 132-5.

45. Meyer-Tuve A, Malcangio M, Ebersberger A, Mazario J, Schaible HG. Effect of brain-derived neurotrophic factor on the release of substance $P$ from rat spinal cord. Neuroreport $2001 ; 12: 21-4$ 